OPEN ACCESS

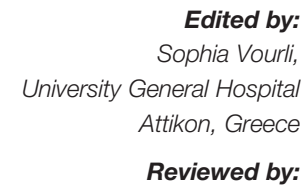

Tatiana Castro Abreu Pinto,

Federal University of Rio de

Janeiro, Brazil

Gino Mongelli,

Centro Neurolesi Bonino Pulejo (IRCCS), Italy

*Correspondence:

Jennifer A. Gaddy

jennifer.a.gaddy@vanderbilt.edu

Steven D. Townsend

steven.d.townsend@vanderbilt.edu

Specialty section:

This article was submitted to Clinical Microbiology,

a section of the journal

Frontiers in Cellular and

Infection Microbiology

Received: 13 July 2021 Accepted: 01 September 2021 Published: 20 September 2021

Citation:

$L u$ J, Guevara MA, Francis JD, Spicer SK, Moore RE, Chambers SA, Craft KM, Manning SD, Townsend SD and Gaddy JA (2021) Analysis of Susceptibility to the Antimicrobial and Anti-Biofilm Activity of Human Milk

Lactoferrin in Clinical Strains of

Streptococcus agalactiae With Diverse Capsular and Sequence Types. Front. Cell. Infect. Microbiol. 11:740872. doi: 10.3389/fcimb.2021.740872

\section{Analysis of Susceptibility to the Antimicrobial and Anti-Biofilm Activity of Human Milk Lactoferrin in Clinical Strains of Streptococcus agalactiae With Diverse Capsular and Sequence Types}

Jacky Lu ${ }^{1}$, Miriam A. Guevara ${ }^{1}$, Jamisha D. Francis ${ }^{1}$, Sabrina K. Spicer ${ }^{2}$, Rebecca E. Moore ${ }^{2}$, Schuyler A. Chambers ${ }^{2}$, Kelly M. Craft ${ }^{3}$, Shannon D. Manning ${ }^{4}$, Steven D. Townsend ${ }^{2 *}$ and Jennifer A. Gaddy ${ }^{1,5,6 *}$

1 Department of Pathology, Microbiology, and Immunology, Vanderbilt University School of Medicine, Nashville, TN, United States, ${ }^{2}$ Department of Chemistry, Vanderbilt University, Nashville, TN, United States, ${ }^{3}$ Department of Chemistry, Harvard University, Cambridge, MA, United States, ${ }^{4}$ Department of Microbiology and Molecular Genetics, Michigan State University, East Lansing, MI, United States, ${ }^{5}$ Division of Infectious Diseases, Department of Medicine, Vanderbilt University Medical Center, Nashville, TN, United States, ${ }^{6}$ Tennessee Valley Healthcare Systems, Department of Veterans Affairs, Nashville, TN, United States

Group B Streptococcus (GBS) is one of the leading infection-related causes of adverse maternal and neonatal outcomes. This includes chorioamnionitis, which leads to preterm ruptures of membranes and can ultimately result in preterm or stillbirth. Infection can also lead to maternal and neonatal sepsis that may contribute to mortality. Currently, treatment for GBS infection include a bolus of intrapartum antibiotic prophylaxis to mothers testing positive for GBS colonization during late pregnancy. Lactoferrin is an antimicrobial peptide expressed in human breast milk, mucosal epithelia, and secondary granules of neutrophils. We previously demonstrated that lactoferrin possesses antimicrobial and antibiofilm properties against several strains of GBS. This is largely due to the ability of lactoferrin to bind and sequester iron. We expanded upon that study by assessing the effects of purified human breast milk lactoferrin against a panel of phenotypically and genetically diverse isolates of GBS. Of the 25 GBS isolates screened, lactoferrin reduced bacterial growth in 14 and biofilm formation in 21 strains. Stratifying the data, we observed that colonizing strains were more susceptible to the growth inhibition activity of lactoferrin than invasive isolates at lactoferrin concentrations between 250-750 $\mu \mathrm{g} / \mathrm{mL}$. Treatment with $750 \mu \mathrm{g} / \mathrm{mL}$ of lactoferrin resulted in differences in bacterial growth and biofilm formation between discrete sequence types. Differences in bacterial growth were also observed between capsular serotypes 1a and III. Maternally isolated strains were more susceptible to lactoferrin with respect to bacterial growth, but not biofilm formation, compared to neonatal sepsis isolates. Finally, high biofilm forming GBS strains were more 
impacted by lactoferrin across all isolates tested. Taken together, this study demonstrates that lactoferrin possesses antimicrobial and antibiofilm properties against a wide range of GBS isolates, with maternally isolated colonizing strains being the most susceptible.

Keywords: biofilm, lactoferrin, Streptococcus agalactiae, nutritional immunity, antimicrobial

\section{INTRODUCTION}

Streptococcus agalactiae, more commonly known as Group B Streptococcus (GBS), is amongst the leading infection-related causes of adverse pregnancy and neonatal outcomes (Shabayek and Spellerberg, 2018). Adverse maternal complications include chorioamnionitis, preterm premature rupture of membranes (PPROM), preterm birth, stillbirth, and maternal sepsis (Goldenberg et al., 2008; Koumans et al., 2012). For the newborn, GBS infections can result in early- and late- onset neonatal sepsis, meningitis, and endocarditis. Early onset disease (EOD) occurs in neonates up to a week after birth (Verani et al., 2010). Neonates with EOD usually present with pneumonia and sepsis. In contrast, late onset disease (LOD) defines infection between 1-week and 3 months after birth and most commonly manifests as sepsis and meningitis. Newborns who survive LOD frequently suffer from neurodevelopmental impairments (Russell et al., 2017).

GBS is a gram-positive encapsulated bacterium, and a commensal member of the human microflora in the gastrointestinal tract. While GBS asymptomatically colonizes $20-30 \%$ of adults, the bacterium may traverse from the lower gastrointestinal tract to the vagina and infect the neonate through ascending infection or ingestion/inhalation of infectious fluids during childbirth (Verani et al., 2010). Indeed, the primary risk factor for EOD is rectovaginal colonization of pregnant women with GBS during delivery (Seale et al., 2017b). The ability of GBS to colonize and persist in the maternal urogenital tract to cause disease is related to its ability to form biofilms (Rosini and Margarit, 2015). Colonization rates differ worldwide, spanning between 6.5-36\% (Shabayek and Spellerberg, 2018). Some recent reports include colonization rates of $13.2 \%$ in a cohort in Ethiopia (Ali et al., 2020), 16.6\% in the Western Cape region of South Africa (Africa and Kaambo, 2018), and $21.6 \%$ over a twelve-year span in North Carolina, USA (Edwards et al., 2019).

GBS strains can be divided into 10 distinct serotypes (Ia, Ib, and II to IX) based on a serological reaction directed against the polysaccharide capsule (Teatero et al., 2017). The streptococcal polysaccharide capsule facilitates evasion of the innate immune response by protecting the bacterial cell from deposition of complement, opsonization, and phagocytosis (Winkelstein et al., 1980; Brown et al., 1983; Abeyta et al., 2003). A recent study from our laboratory revealed that the GBS capsule aids in biofilm formation and ascending infection of the reproductive tract during pregnancy (Noble et al., 2021). Moreover, the capsule across all serotypes shares terminal sialic acid (Sia) residues that allow molecular mimicry of human cell surface sialic acids. This allows interaction with Sia-receptors, Siglecs, on innate immune cells that serve to dampen inflammatory responses (Carlin et al., 2007). Different capsular serotypes result in different range and severity in human disease. For instance, capsular serotype III strains are associated with higher rates of invasive neonatal disease (Alhhazmi et al., 2017) and account for the majority of late-onset meningitis cases in neonates (Bellais et al., 2012). In contrast, serotype Ia and $\mathrm{V}$ predominate among invasive isolates in non-pregnant cases (Phares et al., 2008). However, dominate serotypes fluctuate between regions and across time (Shabayek and Spellerberg, 2018).

Another method by which GBS strains are grouped and characterized is multi-locus sequence typing (MLST), which classifies strains into sequence types (STs) based on allelic variation within seven conserved housekeeping genes (Jones et al., 2003). Based on phylogenetic analysis, GBS STs can be grouped into multiple clonal complexes (CCs) with most human isolates belonging to CC1, CC10, CC17, CC19, CC23, and CC26 (Sørensen et al., 2010). Similar to capsular type, ST diversity also manifests in different disease outcomes and severities. For instance, a study with GBS from multiple continents revealed that STs 1 and 19 have been linked to asymptomatic colonization, while ST-17 predominately related to invasive neonatal disease. ST-23 was associated with both carriage and invasive GBS disease (Jones et al., 2003). All four STs, however, were found to colonize pregnant women at higher rates in different patient populations (Manning et al., 2009). ST-17 serotype III, alongside ST-291, belong to CC17, a group of GBS noted for its hypervirulence (Bellais et al., 2012). ST-17 is strongly linked to both EOD and LOD, as well as meningitis (Musser et al., 1989; Lin et al., 2006; Manning et al., 2008). Meanwhile, ST-291 belonging to serotype IV is strongly associated with EOD and septicemia (Tien et al., 2011; Wang et al., 2015).

In order to combat GBS, the immune system deploys a repertoire of antimicrobial peptides. These peptides aid in combatting infection through the process of nutritional immunity, or the sequestration of essential metals to starve bacteria (Hood and Skaar, 2012). Bacteria require these trace elements as cofactors for essential biological processes. One important example of a protein expressed in defense against GBS is lactoferrin (Kothary et al., 2017; Lu et al., 2020). Lactoferrin is a glycoprotein that contains two iron binding sites (Weinberg, 1975) and was shown to have antimicrobial activity against a wide range of bacterial, viral, and fungal pathogens ( $\mathrm{Lu}$ et al., 2020). Indeed, our previous study demonstrated that human breast milk lactoferrin has antimicrobial and anti-biofilm activity against GBS and inhibits some GBS strains from adhering to human gestational membranes (Lu et al., 2021). In this study, we advanced our findings by analyzing the antimicrobial and anti-biofilm effects of lactoferrin 
against a larger panel of clinical GBS strains that vary by capsular serotype, ST, isolation source, and clinical presentation. We observed broad antimicrobial and antibiofilm action by lactoferrin against most GBS strains, though the maternal colonizing strains were more susceptible to inhibitory effects than the neonatal invasive strains.

\section{MATERIALS AND METHODS}

\section{Bacterial Strains and Culture Conditions}

This study utilized a diverse set of 25 previously characterized $S$. agalactiae strains recovered from neonates with invasive disease (Manning et al., 2009) and colonized mothers sampled before and after childbirth (Manning et al., 2008); all strains were originally isolated by Dr. H. Dele Davies (Davies et al., 2001; Spaetgens et al., 2002). GBS strains were cultured on tryptic soy agar plates supplemented with $5 \%$ sheep blood (blood agar plates) at $37^{\circ} \mathrm{C}$ in ambient air overnight. Bacteria were sub-cultured from blood agar plates into liquid medium (Todd Hewitt Broth; THB) and incubated in aerobic conditions (ambient air, shaking at $200 \mathrm{rpm}$ ) at $37^{\circ} \mathrm{C}$ overnight. The following day, bacterial density was measured spectrophotometrically to determine the optical density at $600 \mathrm{~nm}\left(\mathrm{OD}_{600}\right)$. These bacterial cultures were used for growth, viability, biofilm, and co-culture assays.

\section{Purification of Lactoferrin From Human Breast Milk}

Human lactoferrin was isolated from breast milk as previously described (Lu et al., 2021). Briefly, expressed human breast milk was gathered from 17 healthy donors between 3 days and 3 months post-partum and stored between -80 and $-20^{\circ} \mathrm{C}$. De-identified human milk samples were provided by Dr. J. Hendrik Weitkamp from the Vanderbilt Department of Pediatrics, under a collection protocol approved by the Vanderbilt University Institutional Review Board (IRB \#100897). Milk samples were thawed and centrifuged at $8000 \mathrm{~g}$ for 45 minutes to separate milk fats from the soluble fraction. Following centrifugation, the resultant top lipid layer was removed. Subsequently, proteins were precipitated from the soluble fraction by the addition of ammonium sulfate to the soluble fraction and incubation at $4^{\circ} \mathrm{C}$ overnight. Precipitated proteins were fractionated by ion-exchange chromatography. Cation exchange (CM Sephadex C-50, GE Healthcare) resin suspension was packed in a column $(300 \times 18 \mathrm{~mm})$. After sample loading, the column was washed with equilibration buffer until the absorbance at $280 \mathrm{~nm}$ was less than 0.05 . The bound protein was then displaced from the resin by a stepwise elution protocol. For elution, $10 \mathrm{mM}$ sodium phosphate buffer containing $0.4 \mathrm{M} \mathrm{NaCl}, 0.6$ $\mathrm{M} \mathrm{NaCl}$ and $0.8 \mathrm{M} \mathrm{NaCl}$ were used as elution buffer $\mathrm{A}, \mathrm{B}$, and $\mathrm{C}$, respectively. First, elution buffer A was passed through the column. $5 \mathrm{~mL}$ fractions were collected and the $\mathrm{OD}_{280}$ value of each fraction was measured by a UV-vis spectrophotometer. The elution was continued until the fractions showed a minimum OD of 0.03 . Further elution of the bound protein was carried out with elution buffer B and C. The Identity of the fractions were determined by high resolution mass spectrometry analysis. Fractions containing greater than 99\% lactoferrin were combined and used in the assays. All lactoferrin used in this study was in the apo-form.

\section{Evaluation of Bacterial Growth}

Bacterial growth was determined by a spectrophometric reading as previously described (Lu et al., 2021). Briefly, optical density measurements at $600 \mathrm{~nm}\left(\mathrm{OD}_{600}\right)$ were recorded to determine bacterial growth. GBS cultures were grown to stationary phase $\left(\mathrm{OD}_{600}\right.$ between 0.2-0.3) and diluted at 1:10 in metal-limited THB medium (50\% THB with $50 \%$ calprotectin buffer [ $100 \mathrm{mM} \mathrm{NaCl}, 3$ $\mathrm{mM} \mathrm{CaCl}_{2}, 20 \mathrm{mM}$ Tris pH 7.5 (Senkovich et al., 2010; Haley et al., 2015)]. $100 \mu \mathrm{L}$ of 1:10 diluted cultures were added to each well in a 96-well plate. The appropriate concentration of purified lactoferrin $(0,250,500,750$, or $1000 \mu \mathrm{g} / \mathrm{mL}$, concentrations which are physiologically relevant to the host-pathogen in vivo environment) was added into each corresponding well. The plates were incubated at $37^{\circ} \mathrm{C}$ overnight. The following day, bacterial density was determined by measuring $\mathrm{OD}_{600}$.

\section{Quantification of Bacterial Biofilms}

A crystal violet assay was utilized to evaluate bacterial biofilms as previously described (Gaddy et al., 2009; Lu et al., 2021). Briefly, overnight GBS cultures were diluted 1:10 in THB-CP medium in 96-well plates. To analyze the effect of lactoferrin on biofilm inhibition, lactoferrin was applied in increasing concentrations $(0,250,500,750$, or $1000 \mu \mathrm{g} / \mathrm{mL})$ at the time of inoculation. Biofilms were allowed to form at $37^{\circ} \mathrm{C}$ in ambient air overnight. $\mathrm{OD}_{600}$ was determined using a spectrophotometer and supernatant was removed and replaced with $0.1 \%$ crystal violet stain for thirty minutes. Wells were washed with deionized water three times and dried. The retained crystal violet was resolubilized with a solution of $80 \%$ ethanol and $20 \%$ acetone. Plates were incubated for at least 30 minutes and optical density was determined at $560 \mathrm{~nm}\left(\mathrm{OD}_{560}\right)$. Quantification was determined by using a ratio of $\mathrm{OD}_{560} / \mathrm{OD}_{600}$.

\section{Statistical Analyses}

Statistical analyses of biofilm formation and bacterial growth were performed using Student's t-test or a one-way ANOVA with either Tukey's or Dunnett's post hoc correction for multiple comparisons. All reported $P$ values are adjusted to account for multiple comparisons. $P$ values of $\leq 0.05$ were considered significant. All data analyzed in this work were derived from at least three biological replicates, data points reflect mean of technical replicates (1-3 technical replicates per biological replicate). Statistical analyses were performed using GraphPad Prism software (Versions 6 and 9, GraphPad Prism Software Inc., La Jolla, California).

\section{RESULTS}

\section{Human Breast Milk Lactoferrin Suppresses Bacterial Growth in Many Clinical GBS Isolates}

We previously reported that human breast milk lactoferrin possesses antimicrobial activity against three clinical isolates of 
GBS (Lu et al., 2021). To enhance the generalizability of these findings, we increased the number of GBS strains, thereby capturing more isolates across diverse capsular serotypes and STs. We investigated the effects of lactoferrin treatment across increasing concentrations against this panel of clinical isolates. Out of the 25 GBS strains screened, four strains exhibited inhibition of bacterial growth when treated with $250 \mu \mathrm{g} / \mathrm{mL}$ of human lactoferrin (Table 1; $\mathrm{P}<0.05$, Student's t-test). Growth of 9 total strains was inhibited when treated with a concentration of lactoferrin of $500 \mu \mathrm{g} / \mathrm{mL}$ ( $\mathrm{P}<0.05$, Student's $t$-test $)$. At $750 \mu \mathrm{g} / \mathrm{mL}$ of lactoferrin, 14 strains exhibited a decrease in bacterial growth as compared to media only ( $\mathrm{P}<0.05$, Student's $t$-test). Finally, the growth of 14 strains was inhibited when treated with $1,000 \mu \mathrm{g} / \mathrm{mL}$ of lactoferrin $(\mathrm{P}<0.05$, Student's $t$-test). While there was a trending decrease in bacterial growth for 10 strains relative to the media control, the differences were not statistically significant. No significant differences in growth in medium alone were observed across diverse strains of GBS.

\section{Human Breast Milk Lactoferrin Exhibits Anti-Biofilm Activity Against Numerous Clinical GBS Strains}

Because we previously described the iron-dependent anti-biofilm properties of lactoferrin against a limited number of GBS strains (Lu et al., 2021), we expanded the number of GBS strains to determine if lactoferrin can suppress other strains with diverse genetic backgrounds. Among the 25 strains assayed, 20 strains exhibited a significant decrease in biofilm formation when treated with $250 \mu \mathrm{g} /$ $\mathrm{mL}$ (Table 1; $\mathrm{P}<0.05$, Student's t-test). When the concentration of lactoferrin was increased to $500 \mu \mathrm{g} / \mathrm{mL}$, one additional strain showed susceptibility and a decrease in biofilm production. Of all the strains screened, only four had no reduction in biofilm formation across increasing concentrations of human lactoferrin.

\section{Colonizing GBS Strains Are More Susceptible to Lactoferrin Than Invasive Isolates}

GBS strains were grouped into two groups - colonizing and invasive - and susceptibility to lactoferrin was compared to GBS grown in medium alone without lactoferrin supplementation. At $250 \mu \mathrm{g} / \mathrm{mL}$, the 13 colonizing strains were more susceptible to lactoferrin compared to the 12 invasive strains in respect to bacterial growth with a $12.21 \%$ vs $1.76 \%$ mean reduction, respectively (Figure 1A; $\mathrm{P}<0.05$, Student's t-test). Increasing the concentration of lactoferrin to $500 \mu \mathrm{g} / \mathrm{mL}$ resulted in enhanced suppression of bacterial growth in the colonizing versus invasive strains $(28.14 \%$ vs $17.48 \%$ mean reduction, respectively) ( $\mathrm{P}<0.01$, Student's t-test). Although the addition of $750 \mu \mathrm{g} / \mathrm{mL}$ of lactoferrin decreased bacterial growth for both types of strains, the invasive strains were more resistant to inhibition than the colonizing strains $(20.71 \%$ vs $33.17 \%$ mean reduction, respectively) ( $\mathrm{P}<0.01$, Student's t-test). Lactoferrin at $1,000 \mu \mathrm{g} / \mathrm{mL}$ asserted antimicrobial activity against both types of strains but no differences were observed between the two strain types. With respect to biofilm formation, only the addition of lactoferrin at $250 \mu \mathrm{g} / \mathrm{mL}$ inhibited biofilm formation in the colonizing strains more than invasive ones $(33.25 \%$ vs $22.01 \%$ mean reduction, respectively) (Figure 1B; $\mathrm{P}<0.05$, Student's t-test).

TABLE 1 | Isolation source, capsular type, and sequence type (ST) of clinical strains of Streptococcus agalactiae used in this study and the minimum inhibitory concentration ( $\mathrm{MIC}$ ) of lactoferrin required to suppress growth (as determined by $\mathrm{OD}_{600}$ ) and biofilm (as determined by $\mathrm{OD}_{560} / \mathrm{OD}_{600}$ ).

\begin{tabular}{|c|c|c|c|c|c|c|}
\hline Strain Number & Strain Type & Sequence Type & Capsular Serotype & Isolation Source & Growth MIC & Biofilm MIC \\
\hline GB0002 & Colonizing & ST-23 & cpsla & Vaginal/rectal colonization & $250 \mu \mathrm{g} / \mathrm{mL}$ & $250 \mu \mathrm{g} / \mathrm{mL}$ \\
\hline GB0012 & Colonizing & ST-1 & cpsV & Vaginal/rectal colonization & $750 \mu \mathrm{g} / \mathrm{mL}$ & $250 \mu \mathrm{g} / \mathrm{mL}$ \\
\hline GB0037 & Invasive & ST-1 & cpsV & EOD/sepsis & $>1000 \mu \mathrm{g} / \mathrm{mL}$ & $250 \mu \mathrm{g} / \mathrm{mL}$ \\
\hline GB0064 & Invasive & ST-17 & cpslll & EOD/sepsis & $>1000 \mu \mathrm{g} / \mathrm{mL}$ & $500 \mu \mathrm{g} / \mathrm{mL}$ \\
\hline GB0066 & Invasive & ST-19 & cpsill & EOD/sepsis & $500 \mu \mathrm{g} / \mathrm{mL}$ & $>1000 \mu \mathrm{g} / \mathrm{mL}$ \\
\hline GB0069 & Invasive & ST-17 & cpslll & EOD/sepsis & $500 \mu \mathrm{g} / \mathrm{mL}$ & $>1000 \mu \mathrm{g} / \mathrm{mL}$ \\
\hline GB0079 & Invasive & ST-19 & cpslll & EOD/sepsis & $>1000 \mu \mathrm{g} / \mathrm{mL}$ & $250 \mu \mathrm{g} / \mathrm{mL}$ \\
\hline GB0083 & Colonizing & ST-1 & cpsVI & Vaginal/rectal colonization & $>1000 \mu \mathrm{g} / \mathrm{mL}$ & $250 \mu \mathrm{g} / \mathrm{mL}$ \\
\hline GB0112 & Colonizing & ST-12 & cpslll & Vaginal/rectal colonization & 500 g/mL & $250 \mu \mathrm{g} / \mathrm{mL}$ \\
\hline GB0115 & Colonizing & ST-17 & cpsill & Vaginal/rectal colonization & $250 \mu \mathrm{g} / \mathrm{mL}$ & $<1000 \mu \mathrm{g} / \mathrm{mL}$ \\
\hline GB0241 & Colonizing & ST-23 & cpsV & Vaginal/rectal colonization & $>1000 \mu \mathrm{g} / \mathrm{mL}$ & $250 \mu \mathrm{g} / \mathrm{mL}$ \\
\hline GB0285 & Colonizing & ST-12 & cpsll & Vaginal/rectal colonization & $750 \mu \mathrm{g} / \mathrm{mL}$ & $250 \mu \mathrm{g} / \mathrm{mL}$ \\
\hline GB0291 & Colonizing & ST-12 & cpsll & Vaginal/rectal colonization & 500 rg/mL & $250 \mu \mathrm{g} / \mathrm{mL}$ \\
\hline GB0374 & Invasive & ST-12 & cpslb & EOD/sepsis & $>1000 \mu \mathrm{g} / \mathrm{mL}$ & $250 \mu \mathrm{g} / \mathrm{mL}$ \\
\hline GB0377 & Invasive & ST-19 & cpslll & EOD/sepsis & $>1000 \mu \mathrm{g} / \mathrm{mL}$ & $250 \mu \mathrm{g} / \mathrm{mL}$ \\
\hline GB0390 & Invasive & ST-23 & cpsla & EOD/sepsis & $>1000 \mu \mathrm{g} / \mathrm{mL}$ & $250 \mu \mathrm{g} / \mathrm{mL}$ \\
\hline GB0397 & Invasive & ST-23 & cpslll & EOD/sepsis & $750 \mu \mathrm{g} / \mathrm{mL}$ & $250 \mu \mathrm{g} / \mathrm{mL}$ \\
\hline GB0411 & Invasive & ST-17 & cpslll & EOD/sepsis & $750 \mu \mathrm{g} / \mathrm{mL}$ & $<1000 \mu \mathrm{g} / \mathrm{mL}$ \\
\hline GB0418 & Invasive & ST-17 & cpslll & EOD/sepsis & 750 g/mL & $250 \mu \mathrm{g} / \mathrm{mL}$ \\
\hline GB0438 & Invasive & ST-12 & cpslb & LOD/sepsis & $>1000 \mu \mathrm{g} / \mathrm{mL}$ & $250 \mu \mathrm{g} / \mathrm{mL}$ \\
\hline GB0571 & Colonizing & ST-19 & cpslll & Vaginal/rectal colonization & $250 \mu \mathrm{g} / \mathrm{mL}$ & $250 \mu \mathrm{g} / \mathrm{mL}$ \\
\hline GB0590 & Colonizing & ST-19 & cpslll & Vaginal/rectal colonization & $>1000 \mu \mathrm{g} / \mathrm{mL}$ & $250 \mu \mathrm{g} / \mathrm{mL}$ \\
\hline GB0653 & Colonizing & ST-12 & cpsll & Vaginal/rectal colonization & 500 g/mL & $250 \mu \mathrm{g} / \mathrm{mL}$ \\
\hline GB0654 & Colonizing & ST-17 & cpsill & Vaginal/rectal colonization & $250 \mu \mathrm{g} / \mathrm{mL}$ & $250 \mu \mathrm{g} / \mathrm{mL}$ \\
\hline GB0663 & Colonizing & ST-19 & cpslll & Vaginal/rectal colonization & $>1000 \mu \mathrm{g} / \mathrm{mL}$ & $250 \mu \mathrm{g} / \mathrm{mL}$ \\
\hline
\end{tabular}



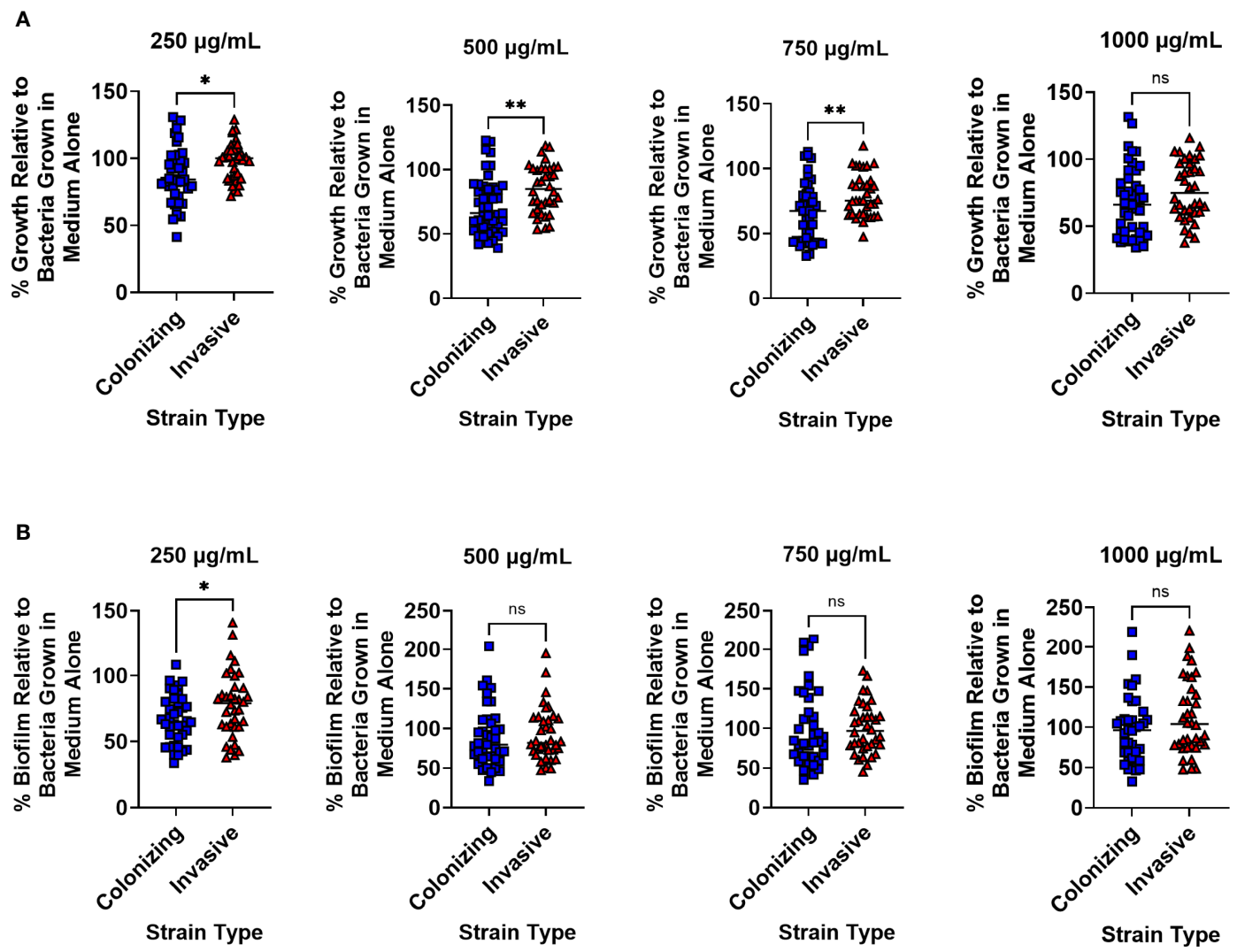

FIGURE 1 | Analysis of susceptibility to lactoferrin-associated growth or biofilm inhibition in invasive vs. clinical isolates of Group B Streptococcus (GBS). GBS strains isolated from colonized patients, or patients experiencing invasive disease were grown in medium alone or increasing concentrations of lactoferrin. (A) Bacterial growth was measured at $24 \mathrm{hr}$ post-inoculation and percent growth was calculated with reference to growth observed in the medium alone negative control. At 250, 500, and $750 \mu \mathrm{g} / \mathrm{mL}$, colonizing strains of GBS (blue squares) exhibited greater growth inhibition than invasive strains (red triangles) as determined by Student's t-test with Welch's correction ( ${ }^{*} \mathrm{P}<0.05$, and ${ }^{* *} \mathrm{P}<0.01$ ). (B) Bacterial biofilm was measured at 24 hr post-inoculation and percent growth was calculated with reference to growth observed in the medium alone negative control. At $250 \mu \mathrm{g} / \mathrm{mL}$, colonizing strains of GBS (blue squares) exhibited greater biofilm inhibition than invasive strains (red triangles) as determined by Student's t-test with Welch's correction ( $\left.{ }^{\star} \mathrm{P}<0.05\right)$. ns, not statistically significant.

\section{Treatment With Human Lactoferrin at $750 \mu \mathrm{g} / \mathrm{mL}$ Reveals Differences in Susceptibility Between GBS Sequence Types (STs)}

Because different GBS STs are associated with maternal colonization and neonatal disease, it is possible that different strains have variable mechanisms to cope with iron starvation. To investigate this possibility, GBS strains were binned by ST and susceptibility to lactoferrin was analyzed between STs. No significant differences in bacterial growth were detected with treatment at 250,500 , and $1,000 \mu \mathrm{g} / \mathrm{mL}$ of lactoferrin between the different STs; however, differences were observed with 750 $\mu \mathrm{g} / \mathrm{mL}$ of lactoferrin. Specifically, the ST-1 strains were more resistant to bacterial growth suppression compared to ST-12 strains (Figure 2A; $\mathrm{P}<0.05$, One-way ANOVA; post hoc Tukey's test). Similar differences in biofilm suppression were observed between STs while treating with $750 \mu \mathrm{g} / \mathrm{mL}$ of lactoferrin. At this concentration, the ST-17 strains were more resistant to the antibiofilm activity of lactoferrin compared to both ST-19 and
ST-23 strains (Figure $2 B ; \mathrm{P}<0.01$ and $\mathrm{P}<0.05$, respectively, One-way ANOVA; post hoc Tukey's test).

\section{Treatment With Human Lactoferrin at $250 \mu \mathrm{g} / \mathrm{mL}$ Reveals Differences in Susceptibility Across Capsular Types}

It is plausible that capsular type may also influence resistance to lactoferrin. To test this, strains were grouped into cohorts based on capsular serotype and susceptibility to lactoferrin across capsule types were analyzed. No significant differences in resistance against the antimicrobial activity of lactoferrin were observed between capsular types across increasing concentrations of lactoferrin (Figure 3A; P > 0.05, One-way ANOVA; post hoc Tukey's test). However, treatment with lactoferrin at $250 \mu \mathrm{g} / \mathrm{mL}$ showed that capsular type III strains exhibited resistance to its antibiofilm activity compared to capsular type 1a isolates (Figure 3B; $\mathrm{P}<$ 0.05 , One-way ANOVA; post hoc Tukey's test). This phenotype was ablated with the additional stress imposed by increasing concentrations of lactoferrin. 

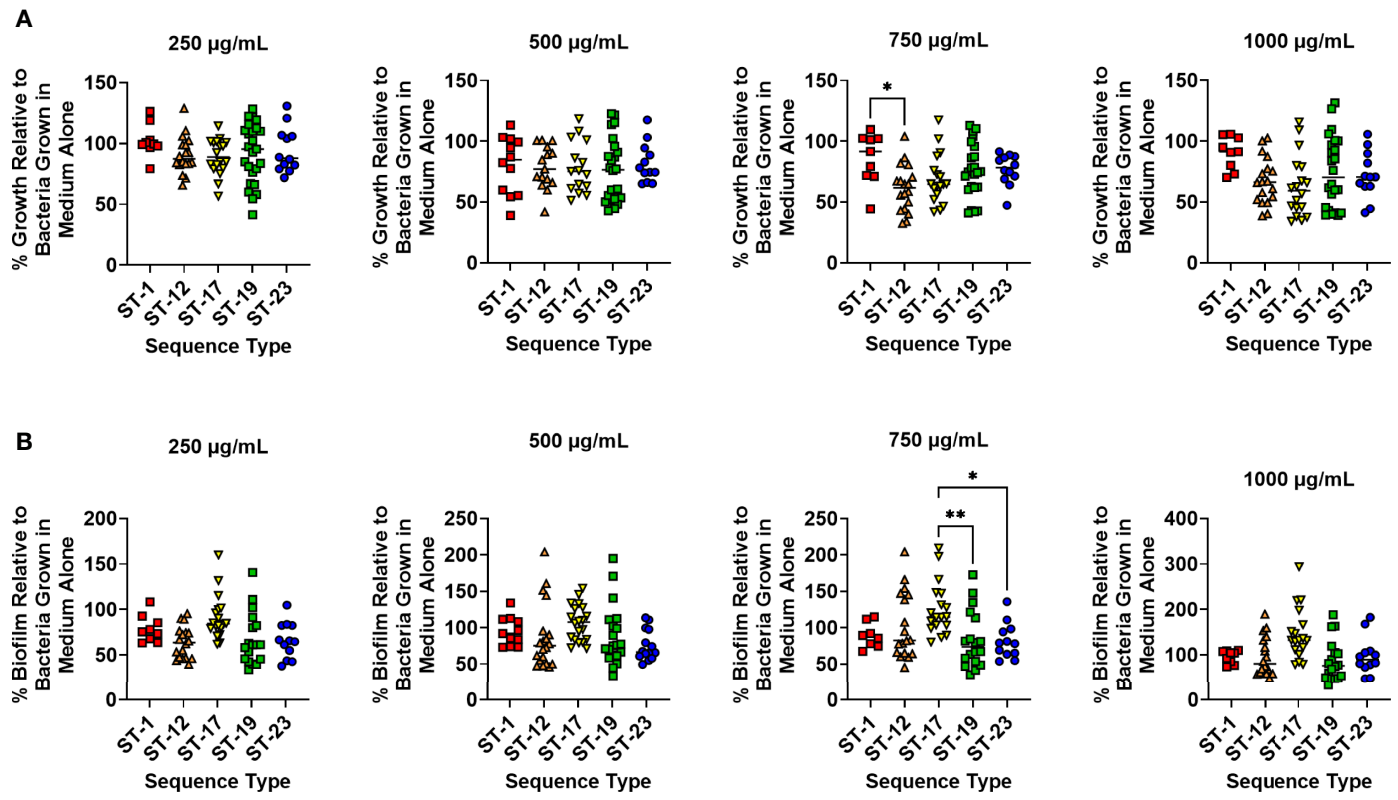

FIGURE 2 | Analysis of susceptibility to lactoferrin-associated growth inhibition in diverse sequence types (STs) of Group B Streptococcus (GBS). GBS strains isolated with a variety of sequence type (ST-1, red; ST-12, orange; ST-17, yellow; ST-19, green; ST-23, blue) were grown in medium alone or increasing concentrations of lactoferrin. (A) Bacterial growth was measured at $24 \mathrm{hr}$ post-inoculation and percent growth was calculated with reference to growth observed in the medium alone negative control. At $750 \mu \mathrm{g} / \mathrm{mL}$, ST-12 strains of GBS exhibited greater growth inhibition than ST-1 strains as determined by one-way ANOVA with post hoc Tukey's test ( $\left.{ }^{\star} \mathrm{P}<0.05\right)$. (B) Bacterial biofilm was measured at $24 \mathrm{hr}$ post-inoculation and percent biofilm was calculated with reference to biofilm observed in the medium alone negative control. At $750 \mu \mathrm{g} / \mathrm{mL}$, ST-19 and ST-23 strains of GBS exhibited greater biofilm inhibition than ST-17 strains as determined by one-way ANOVA with post hoc Tukey's test ( ${ }^{*} P<0.05$, and ${ }^{* *} P<0.01$ ).

\section{Lactoferrin Asserts Anti-Biofilm Effects Against Both High and Low Biofilm Formers but Enhances Biofilm Formation in Low Biofilm Formers at Higher Concentrations}

Because our group has previously observed a range of biofilm production across GBS strains (Parker et al., 2016), we sought to determine the geometric mean of biofilm produced by all isolates investigated in this study. GBS strains that form biofilms above the determined geometric mean $\left(\mathrm{OD}_{560} /_{600}=0.3965\right)$ were designated as "high" biofilm formers, while those below were named "low". Treatment with $250 \mu \mathrm{g} / \mathrm{mL}$ of lactoferrin significantly inhibited biofilm formation in both high and low biofilm formers (Figure 4; $\mathrm{P}<0.0001$, Student's t-test; $\mathrm{P}<0.001$, Student's t-test, respectively), whereas $500 \mu \mathrm{g} / \mathrm{mL}$ of lactoferrin only inhibited the high biofilm formers $(\mathrm{P}<0.0001$, Student's $\mathrm{t}-$ test). When treated with $750 \mu \mathrm{g} / \mathrm{mL}$ of lactoferrin, the high biofilm formers exhibited a decrease in biofilm formation $(\mathrm{P}<$ 0.05 , Student's t-test), however, the low biofilm formers (mean = 0.3183 ) showed increased biofilm production $($ mean $=0.3501)(\mathrm{P}$ $<0.05$, Student's t-test). This discrepancy between high and low biofilm formers was further amplified at treatment with $1000 \mu \mathrm{g} /$ $\mathrm{mL}$ of lactoferrin $($ mean $=0.3934)(\mathrm{P}<0.001$, Student's t-test $)$.

\section{DISCUSSION}

In this present study, we expanded upon our previous work by increasing the panel of GBS strains to include phenotypically and genetically diverse clinical strains from diverse anatomical sites of isolation and assessing susceptibility to the antimicrobial and anti-biofilm activity of human milk lactoferrin. We discovered that lactoferrin possesses antimicrobial and antibiofilm properties against many diverse GBS strains. In particular, colonizing maternal strains were more susceptible to lactoferrin, compared to invasive neonatal strains.

Other studies have revealed that lactoferrin may contribute to improvement of reproductive tract infections and subsequent disease. For instance, vaginal lactoferrin supplementation in pregnant people with bacterial vaginosis reduced the rate of preterm birth (Miranda et al., 2019). Furthermore, other groups have identified lactoferrin as a critical component of cervicomucosal defense against a variety of lower genital tract infections caused by Neisseria gonorrhoeae, Chlamydia trachomatis, and Trichomonas vaginalis (Pino et al., 2017). One plausible explanation for this phenomenon may be that lactoferrin protects by repressing Gardnerella, Prevotella, and Lachnospira species within the host microbiome, thus decreasing competition for Lactobacillus species and allowing them to 

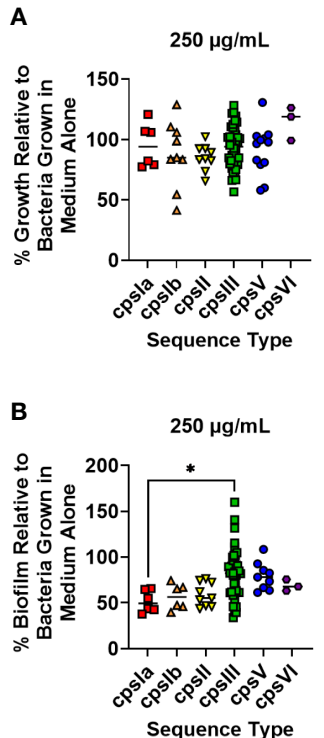
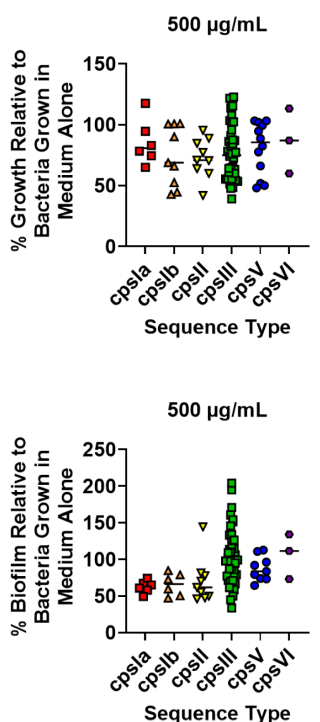
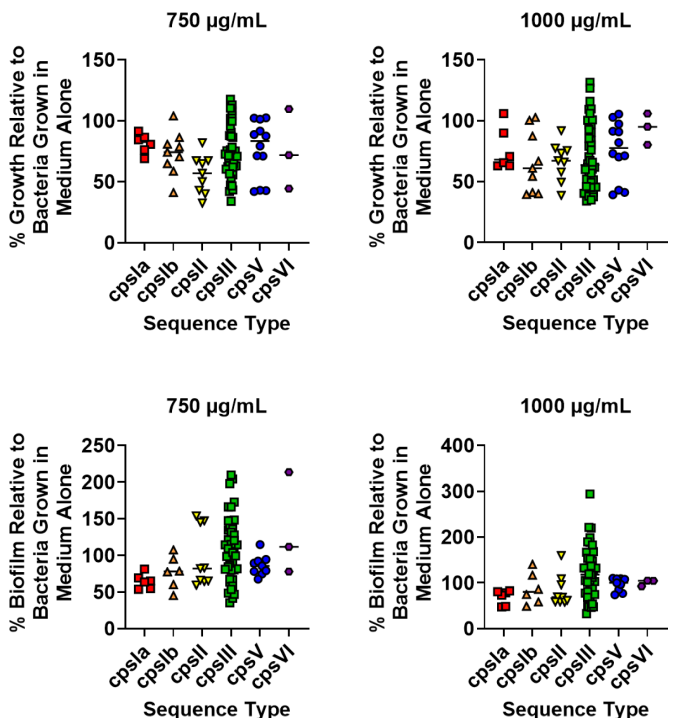

FIGURE 3 | Analysis of susceptibility to lactoferrin-associated growth or biofilm inhibition in diverse capsular serotypes of Group B Streptococcus (GBS). GBS strains isolated with a span of capsular serotypes (cpsla, red; cpslb, orange; cpsll, yellow; cpslll, green; cpsV, blue; cpsVI, purple) were grown in medium alone or increasing concentrations of lactoferrin. (A) Bacterial growth was measured at $24 \mathrm{hr}$ post-inoculation and percent growth was calculated with reference to growth observed in the medium alone negative controls. No differences in growth were detected across molecular serotype as determined by one-way ANOVA with post hoc Tukey's test ( $\left.{ }^{\star} \mathrm{P}<0.05\right)$. (B) Bacterial biofilm formation was measured at $24 \mathrm{hr}$ post-inoculation and percent growth was calculated with reference to growth observed in the medium alone negative controls. At $250 \mu \mathrm{g} / \mathrm{mL}$, cps1a strains of GBS exhibited greater biofilm inhibition than cpslll strains as determined by oneway ANOVA with post hoc Tukey's test ( $\left.{ }^{\star} \mathrm{P}<0.05\right)$.

prevent dysbiosis by dominating the vaginal microbiota (Valenti et al., 2018). Adding credence to this possibility is that Lactobacillus species have been recognized as one of the few bacterial populations that lack a strict nutrient requirement for iron (Imbert and Blondeau, 1998). Thus, it is plausible that the antimicrobial activity of lactoferrin associated with its role in nutritional immunity would be largely ineffective against these important commensals. Indeed, our previous work has shown various Lactobacillus strains and their secreted products modulate GBS interactions with cells of the extraplacental membranes. Specifically, Lactobacillus supernatants inhibited GBS growth, biofilm formation and invasion of host cells, though strain-dependent effects were observed. Notably, supernatant from L. reuteri 6475 broadly inhibited growth in 36 distinct GBS strains and inhibited GBS growth to an average of $46.6 \%$ of each GBS strain alone (Shiroda et al., 2020). Thus, there is merit in studying the use of human lactoferrin alone or in combination with Lactobacillus spp. in the prevention of GBSmediated preterm births and adverse pregnancy outcomes as maternal colonizing GBS strains can infect the fetus by ascending the gravid reproductive tract. Here, we have shown that maternal colonizing GBS strains are greatly susceptible to the antimicrobial and antibiofilm action of lactoferrin.

Our results indicate that certain STs are more susceptible than others at $750 \mu \mathrm{g} / \mathrm{mL}$ of lactoferrin treatment. The MLST scheme uses seven loci that encode enzymes involved in intermediary metabolism to distinguish GBS STs (Jones et al., 2003). Because iron is an important cofactor for many enzymes involved in bacterial metabolism and physiology (Andrews et al., 2003), lactoferrin can help defend against invading bacteria by starving the prokaryotic cells of nutritional iron needed for optimal enzyme activity. However, some enzymes are promiscuous with their utilization of transition metal cofactors. As a result, it is plausible that we witnessed variable antimicrobial effects of lactoferrin because some of these housekeeping enzymes, or another enzyme up- or downstream of its respective pathways, require iron for full function while others may use other transitional metals under conditions of iron starvation (Foster et al., 2014). An alternate explanation is that other enzymes in some pathways with similar functions may be able to compensate for the absence of or limited iron-cofactors. Perhaps the number of loci used in the MSTL were too limited. It is plausible that the effect of lactoferrin is indirect and does not involve the specific genes loci used in the MSTL. Further examination at the whole genome phylogeny may better differentiate lactoferrin effects across close or distantly related genomes regardless of cps type or isolation source.

The capsule of GBS plays an important role in pathogenesis in humans (Vornhagen et al., 2017). As different capsular types have been correlated to varying disease outcomes and susceptibility to antimicrobial agents (Teatero et al., 2017), we hypothesized that different capsular serotypes might have variable responses to the antimicrobial activity of lactoferrin. We observed differences in biofilm formation but not bacterial growth between different GBS 
A

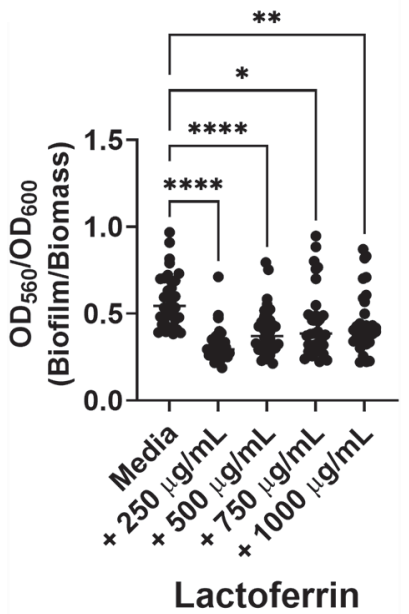

B

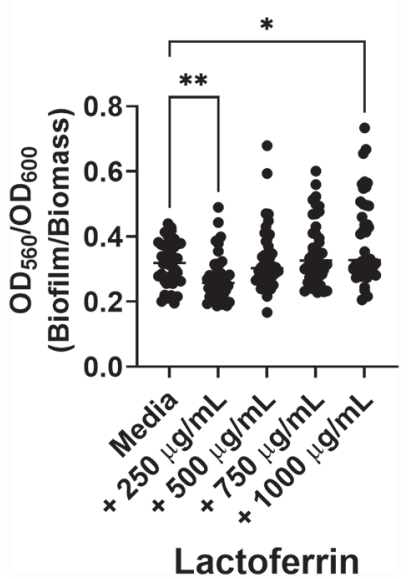

FIGURE 4 | Lactoferrin-dependent inhibition of biofilm based on Group B Streptococcus (GBS) strain variation with respect to high or low biofilm production. GBS biofilm values $\left(\mathrm{OD}_{560}\right)$ were pooled, and the geometric mean was determined. Strains were divided between high (A) and low (B) biofilm formers. High biofilm formers were more susceptible to biofilm inhibition by lactoferrin across all concentrations of lactoferrin. At $250 \mu \mathrm{g} / \mathrm{mL}$, low biofilm forming strains of GBS exhibited inhibition of biofilm formation compared to media alone. However, treatment with $750 \mu \mathrm{g} / \mathrm{mL}$ and $1,000 \mu \mathrm{g} / \mathrm{mL}$ of lactoferrin resulted in an increase in biofilm formation, gas determined by Student's t-test with Welch's correction ${ }^{*} \mathrm{P}<0.05,{ }^{* *} \mathrm{P}<0.01,{ }^{* *} \mathrm{P}<0.001$, and $\left.{ }^{* \star *} P<0.0001\right)$.

capsular types under a lower treatment of lactoferrin. Given that the capsule is an important virulence factor for pathogenesis and evasion of immune assault (Gendrin et al., 2018), it was not surprising that no differences were observed in our controlled in vitro studies free of immune stressors. However, we did observe differences in GBS biofilm formation. Our group previously described the role of capsule in biofilm formation in GBS
(Noble et al., 2021), further bolstering our findings. It is plausible that iron starvation alters capsule-mediated biofilm formation in certain capsular types of GBS. Further in vitro work with host cells or in vivo experiments are needed to bridge our gap in knowledge of these relationships between capsular serotype and lactoferrin.

In our study, we further analyzed differences in biofilm inhibition by lactoferrin between strains that formed robust biofilms compared to those that formed low or weak biofilms. Lactoferrin inhibited biofilm formation in high biofilm forming isolates, which is consistent to with our previous study in a smaller cohort of strains ( $\mathrm{Lu}$ et al., 2021). The intersection between iron and biofilm formation has also been studied in other bacterial pathogens. One study of interest by Trappetti and colleagues described the role of the mononuclear iron protein Sribosylhomocysteine lyase (LuxS) in quorum sensing and biofilm formation in Streptococcus pneumoniae (Trappetti et al., 2011). Consistent with their work, we also observed that iron starvation results in inhibition of biofilm formation. Thus, it is plausible that GBS possesses similar iron-sensing pathways that govern biofilm formation. More work, however, is needed to elucidate and confirm the function of these pathways.

Colonization of the maternal genitourinary tract is the most important risk factor for neonatal GBS disease (Benitz et al., 1999). In a longitudinal study performed by Kwatra and colleagues, up to $50 \%$ of the study cohort was transiently colonized by GBS at some point during pregnancy, highlighting the dynamic nature of GBS colonization (Kwatra et al., 2014). Currently in the United States, pregnant individuals are screened for the presence of GBS between 35 and 37 weeks of gestation (Carrillo-Ávila et al., 2018). If a patient tests positive for GBS, then intrapartum antibiotic prophylaxis (IAP) is administered during labor and delivery to prevent neonatal EOD (Seale et al., 2017a). Though antibiotic treatment is the only current preventative strategy available, the efficacy of IAP against EOD is around $80 \%$ (Fairlie et al., 2013). Despite the availability of IAP, however, the rates of LOD have remained unchanged (Nanduri et al., 2019). Additional drawbacks associated with the use of IAP (Rao and Khanna, 2020) include hypersensitivities to first-line antibiotics (Matteson et al., 2008), alteration of the neonatal microbiota (Nogacka et al., 2017), and emergence of antibiotic resistant strains (Hayes et al., 2020). Hence, the discovery of alternative therapies is critical and may help overcome these IAP drawbacks. Breastfeeding has been associated with protection against infection and could be exploited as a risk-mitigation strategy. However, cases of GBS transfer by breast milk have been recorded (Ager et al., 2020). The protective effects of breast milk are likely derived from the antimicrobial and immunomodulatory molecules that comprise milk, including lactoferrin and human milk oligosaccharides (Andreas et al., 2015). Thus, utilization of these specific molecules could be leveraged to mitigate the risk of GBS transmission. In the study herein, we found that maternal colonizing GBS strains are most susceptible to lactoferrin, suggesting that the antimicrobial peptide may be a viable candidate to aid in the prevention of GBS disease. 


\section{DATA AVAILABILITY STATEMENT}

The original contributions presented in the study are included in the article/supplementary material. Further inquiries can be directed to the corresponding authors.

\section{ETHICS STATEMENT}

Ethics approval to carry out this study was provided by the Vanderbilt University Institutional Review Board (IRB \#100897 for human milk donation).

\section{AUTHOR CONTRIBUTIONS}

KC, JL, and ST purified lactoferrin for the studies. JL performed bacterial culture experiments. SDM curated and validated the clinical strains for use in this study. JL, JF, MG, SC, RM, SS, KC, ST, and JG conceptualized and analyzed results and interpreted

\section{REFERENCES}

Abeyta, M., Hardy, G. G., and Yother, J. (2003). Genetic Alteration of Capsule Type But Not PspA Type Affects Accessibility of Surface-Bound Complement and Surface Antigens of. Streptococcus Pneumoniae Infect. Immun. 71, 218225. doi: 10.1128/iai.71.1.218-225.2003

Africa, C. W. J., and Kaambo, E. (2018). Group B Streptococcus Serotypes in Pregnant Women From the Western Cape Region of South Africa. Front. Public Heal. 6, 356. doi: 10.3389/fpubh.2018.00356

Ager, E. P. C., Steele, E. D., Nielsen, L. E., Nestander, M. A., Mende, K., and Spencer, S. E. (2020). Hypervirulent Streptococcus Agalactiae Septicemia in Twin Ex-Premature Infants Transmitted by Breast Milk: Report of Source Detection and Isolate Characterization Using Commonly Available Molecular Diagnostic Methods. Ann. Clin. Microbiol. Antimicrob. 19, 55. doi: 10.1186/ s12941-020-00396-6

Alhhazmi, A., Pandey, A., and Tyrrell, G. J. (2017). Identification of Group B Streptococcus Capsule Type by Use of a Dual Phenotypic/Genotypic Assay. J. Clin. Microbiol. 55, 2637-2650. doi: 10.1128/JCM.00300-17

Ali, M. M., Asrat, D., Fenta, D. A., Chaka, T. E., and Woldeamanuel, Y. (2020). Group B Streptococcus Colonization Rate and Serotype Distribution Among Pregnant Women and Their Newborns at Adama Hospital Medical College, Ethiopia. Sci. Rep. 10, 9301. doi: 10.1038/s41598-020-66474-Z

Andreas, N. J., Kampmann, B., and Mehring Le-Doare, K. (2015). Human Breast Milk: A Review on its Composition and Bioactivity. Early Hum. Dev. 91, 629635. doi: 10.1016/j.earlhumdev.2015.08.013

Andrews, S. C., Robinson, A. K., and Rodríguez-Quiñones, F. (2003). Bacterial Iron Homeostasis. FEMS Microbiol. Rev. 27, 215-237. doi: 10.1016/S0168-6445 (03)00055-X

Bellais, S., Six, A., Fouet, A., Longo, M., Dmytruk, N., Glaser, P., et al. (2012). Capsular Switching in Group B Streptococcus CC17 Hypervirulent Clone: A Future Challenge for Polysaccharide Vaccine Development. J. Infect. Dis. 206, 1745-1752. doi: 10.1093/infdis/jis605

Benitz, W. E., Gould, J. B., and Druzin, M. L. (1999). Risk Factors for Early-Onset Group B Streptococcal Sepsis: Estimation of Odds Ratios by Critical Literature Review. Pediatrics 103:e77. doi: 10.1542/peds.103.6.e77

Brown, E. J., Joiner, K. A., Cole, R. M., and Berger, M. (1983). Localization of Complement Component 3 on Streptococcus Pneumoniae: Anti-Capsular Antibody Causes Complement Deposition on the Pneumococcal Capsule. Infect. Immun. 39, 403-409. doi: 10.1128/IAI.39.1.403-409.1983

Carlin, A. F., Lewis, A. L., Varki, A., and Nizet, V. (2007). Group B Streptococcal Capsular Sialic Acids Interact With Siglecs (Immunoglobulin-Like Lectins) on Human Leukocytes. J. Bacteriol. 189, 1231-1237. doi: 10.1128/JB.01155-06 data, and wrote and edited the manuscript for critical content. All authors contributed to the article and approved the submitted version.

\section{FUNDING}

This work was funded by the National Institutes of Health grants HD090061 (to JG) and the Department of Veterans Affairs Office of Research BX005352 (to JG). which supported wet bench experiments, 2T32HL007411-39 (to JL), 2T32AI112541-06 (to JF), which supported their time and efforts, National Science Foundation 1847804 which supported reagents and wet bench work. Additional support was provided by the Vanderbilt Institute for Clinical and Translational Research program supported by the National Center for Research Resources, Grant UL1 RR024975-01, and the National Center for Advancing Translational Sciences, Grant 2 UL1 TR000445-06 which supports access to Core Facilities and Biostatistics support.

Carrillo-Ávila, J. A., Gutiérrez-Fernández, J., González-Espín, A. I., GarcíaTriviño, E., and Giménez-Lirola, L. G. (2018). Comparison of qPCR and Culture Methods for Group B Streptococcus Colonization Detection in Pregnant Women: Evaluation of a New qPCR Assay. BMC Infect. Dis. 18, 305. doi: 10.1186/s12879-018-3208-4

Davies, H. D., Adair, C., McGeer, A., Ma, D., Robertson, S., Mucenski, M., et al. (2001). Antibodies to Capsular Polysaccharides of Group B Streptococcus in Pregnant Canadian Women: Relationship to Colonization Status and Infection in the Neonate. J. Infect. Dis. 184, 285-291. doi: 10.1086/322029

Edwards, J. M., Watson, N., Focht, C., Wynn, C., Todd, C. A., Walter, E. B., et al. (2019). Group B Streptococcus (GBS) Colonization and Disease Among Pregnant Women: A Historical Cohort Study. Infect. Dis. Obstet. Gynecol. 2019:5430493. doi: 10.1155/2019/5430493

Fairlie, T., Zell, E. R., and Schrag, S. (2013). Effectiveness of Intrapartum Antibiotic Prophylaxis for Prevention of Early-Onset Group B Streptococcal Disease. Obstet. Gynecol. 121, 570-577. doi: 10.1097/AOG.0b013e318280d4f6

Foster, A. W., Osman, D., and Robinson, N. J. (2014). Metal Preferences and Metallation. J. Biol. Chem. 289, 28095-28103. doi: 10.1074/jbc.R114.588145

Gaddy, J. A., Tomaras, A. P., and Actis, L. A. (2009). The Acinetobacter Baumannii 19606 OmpA Protein Plays a Role in Biofilm Formation on Abiotic Surfaces and in the Interaction of This Pathogen With Eukaryotic Cells. Infect. Immun. 77, 3150-3160. doi: 10.1128/IAI.00096-09

Gendrin, C., Merillat, S., Vornhagen, J., Coleman, M., Armistead, B., Ngo, L., et al. (2018). Diminished Capsule Exacerbates Virulence, Blood-Brain Barrier Penetration, Intracellular Persistence, and Antibiotic Evasion of Hyperhemolytic Group B Streptococci. J. Infect. Dis. 217, 1128-1138. doi: 10.1093/infdis/jix684

Goldenberg, R. L., Culhane, J. F., Iams, J. D., and Romero, R. (2008). Epidemiology and Causes of Preterm Birth. Lancet 371, 75-84. doi: 10.1016/S0140-6736(08)60074-4

Haley, K. P., Delgado, A. G., Piazuelo, M. B., Mortensen, B. L., Correa, P., Damo, S. M., et al. (2015). The Human Antimicrobial Protein Calgranulin C Participates in Control of Helicobacter Pylori Growth and Regulation of Virulence. Infect. Immun. 83 (7), 2944-2956. doi: 10.1128/IAI.00544-15

Hayes, K., O’Halloran, F., and Cotter, L. (2020). A Review of Antibiotic Resistance in Group B Streptococcus: The Story So Far. Crit. Rev. Microbiol. 46, 253-269. doi: 10.1080/1040841X.2020.1758626

Hood, M. I., and Skaar, E. P. (2012). Nutritional Immunity: Transition Metals at the Pathogen-Host Interface. Nat. Rev. Microbiol. 10, 525-537. doi: 10.1038/ nrmicro2836

Imbert, M., and Blondeau, R. (1998). On the Iron Requirement of Lactobacilli Grown in Chemically Defined Medium. Curr. Microbiol. 37, 64-66. doi: $10.1007 /$ s002849900339 
Jones, N., Bohnsack, J. F., Takahashi, S., Oliver, K. A., Chan, M.-S., Kunst, F., et al. (2003). Multilocus Sequence Typing System for Group B Streptococcus. J. Clin. Microbiol. 41, 2530-2536. doi: 10.1128/JCM.41.6.2530-2536.2003

Kothary, V., Doster, R. S., Rogers, L. M., Kirk, L. A., Boyd, K. L., RomanoKeeler, J., et al. (2017). Group B Streptococcus Induces Neutrophil Recruitment to Gestational Tissues and Elaboration of Extracellular Traps and Nutritional Immunity. Front. Cell. Infect. Microbiol. 7, 19. doi: 10.3389/ fcimb.2017.00019

Koumans, E. H. A., Rosen, J., van Dyke, M. K., Zell, E., Phares, C. R., Taylor, A., et al. (2012). Prevention of Mother-to-Child Transmission of Infections During Pregnancy: Implementation of Recommended Interventions, United State-2004. Am. J. Obstet. Gynecol. 206, 158.e1-158.e11. doi: 10.1016/ j.ajog.2011.08.027

Kwatra, G., Adrian, P. V., Shiri, T., Buchmann, E. J., Cutland, C. L., and Madhi, S. A. (2014). Serotype-Specific Acquisition and Loss of Group B Streptococcus Recto-Vaginal Colonization in Late Pregnancy. PloS One 9, e98778. doi: 10.1371/journal.pone.0098778

Lin, F.-Y. C., Whiting, A., Adderson, E., Takahashi, S., Dunn, D. M., Weiss, R., et al. (2006). Phylogenetic Lineages of Invasive and Colonizing Strains of Serotype III Group B Streptococci From Neonates: A Multicenter Prospective Study. J. Clin. Microbiol. 44, 1257-1261. doi: 10.1128/JCM.44.4.1257-1261.2006

Lu, J., Francis, J., Doster, R. S., Haley, K. P., Craft, K. M., Moore, R. E., et al. (2020). Lactoferrin: A Critical Mediator of Both Host Immune Response and Antimicrobial Activity in Response to Streptococcal Infections. ACS Infect. Dis. 6, 1615-1623. doi: 10.1021/acsinfecdis.0c00050

Lu, J., Francis, J. D., Guevara, M. A., Moore, R. E., Chambers, S. A., Doster, R. S., et al. (2021). Antibacterial and Anti-Biofilm Activity of the Human Breast Milk Glycoprotein Lactoferrin Against Group B Streptococcus. Chembiochem. 22 (12), 2124-2133. doi: 10.1002/cbic.202100016

Manning, S. D., Lewis, M. A., Springman, A. C., Lehotzky, E., Whittam, T. S., and Davies, H. D. (2008). Genotypic Diversity and Serotype Distribution of Group B Streptococcus Isolated From Women Before and After Delivery. Clin. Infect. Dis. 46, 1829-1837. doi: 10.1086/588296

Manning, S. D., Springman, A. C., Lehotzky, E., Lewis, M. A., Whittam, T. S., and Davies, H. D. (2009). Multilocus Sequence Types Associated With Neonatal Group B Streptococcal Sepsis and Meningitis in Canada. J. Clin. Microbiol. 47, 1143-1148. doi: 10.1128/JCM.01424-08

Matteson, K. A., Lievense, S. P., Catanzaro, B., and Phipps, M. G. (2008). Intrapartum Group B Streptococci Prophylaxis in Patients Reporting a Penicillin Allergy. Obstet. Gynecol. 111, 356-364. doi: 10.1097/ AOG.0b013e318160ff9d

Miranda, M., Saccone, G., Ammendola, A., Salzano, E., Iannicelli, M., De Rosa, R., et al. (2019). Vaginal Lactoferrin in Prevention of Preterm Birth in Women With Bacterial Vaginosis. J. Matern. Neonatal Med. Off. J. Eur. Assoc. Perinat. Med. Fed. Asia Ocean Perinat. Soc Int. Soc Perinat. Obstet. 13, 1-5. doi: 10.1080/14767058.2019.1690445

Musser, J. M., Mattingly, S. J., Quentin, R., Goudeau, A., and Selander, R. K. (1989). Identification of a High-Virulence Clone of Type III Streptococcus Agalactiae (Group B Streptococcus) Causing Invasive Neonatal Disease. Proc. Natl. Acad. Sci. U. S. A. 86, 4731-4735. doi: 10.1073/pnas.86.12.4731

Nanduri, S. A., Petit, S., Smelser, C., Apostol, M., Alden, N. B., Harrison, L. H., et al. (2019). Epidemiology of Invasive Early-Onset and Late-Onset Group B Streptococcal Disease in The United Stateto 2015: Multistate Laboratory and Population-Based Surveillance. JAMA Pediatr. 173, 224-233. doi: 10.1001/ jamapediatrics.2018.4826

Noble, K., Lu, J., Guevara, M. A., Doster, R. S., Chambers, S. A., Rogers, L. M., et al. (2021). Group B Streptococcus cpsE Is Required for Serotype V Capsule Production and Aids in Biofilm Formation and Ascending Infection of the Reproductive Tract During Pregnancy. ACS Infect. Dis. 7 (9), 2686-2696. doi: 10.1021/acsinfecdis.1c00182

Nogacka, A., Salazar, N., Suárez, M., Milani, C., Arboleya, S., Solís, G., et al. (2017). Impact of Intrapartum Antimicrobial Prophylaxis Upon the Intestinal Microbiota and The Prevalence of Antibiotic Resistance Genes in Vaginally Delivered Full-Term Neonates. Microbiome 5, 93. doi: 10.1186/s40168-0170313-3

Parker, R. E., Laut, C., Gaddy, J. A., Zadoks, R. N., Davies, H. D., and Manning, S. D. (2016). Association Between Genotypic Diversity and Biofilm Production in Group B Streptococcus. BMC Microbiol. 16:86. doi: 10.1186/s12866-016-0704-9
Phares, C. R., Lynfield, R., Farley, M. M., Mohle-Boetani, J., Harrison, L. H., Petit, S., et al. (2008). Epidemiology of Invasive Group B Streptococcal Disease in the United State-2005. JAMA 299, 2056-2065. doi: 10.1001/jama.299.17.2056

Pino, A., Giunta, G., Randazzo, C. L., Caruso, S., Caggia, C., and Cianci, A. (2017). Bacterial Biota of Women With Bacterial Vaginosis Treated With Lactoferrin: An Open Prospective Randomized Trial. Microb. Ecol. Health Dis. 28, 1357417. doi: 10.1080/16512235.2017.1357417

Rao, G. G., and Khanna, P. (2020). To Screen or Not to Screen Women for Group B Streptococcus (Streptococcus Agalactiae) to Prevent Early Onset Sepsis in Newborns: Recent Advances in the Unresolved Debate. Ther. Adv. Infect. Dis. 7, 2049936120942424. doi: 10.1177/2049936120942424

Rosini, R., and Margarit, I. (2015). Biofilm Formation by Streptococcus Agalactiae: Influence of Environmental Conditions and Implicated Virulence Factors. Front. Cell. Infect. Microbiol. 5, 6. doi: 10.3389/fcimb.2015.00006

Russell, N. J., Seale, A. C., O'Sullivan, C., Le Doare, K., Heath, P. T., Lawn, J. E., et al. (2017). Risk of Early-Onset Neonatal Group B Streptococcal Disease With Maternal Colonization Worldwide: Systematic Review and Meta-Analyses. Clin. Infect. Dis. An Off. Publ. Infect. Dis. Soc Am. 65, S152-S159. doi: 10.1093/ cid/cix655

Seale, A. C., Bianchi-Jassir, F., Russell, N. J., Kohli-Lynch, M., Tann, C. J., Hall, J., et al. (2017a). Estimates of the Burden of Group B Streptococcal Disease Worldwide for Pregnant Women, Stillbirths, and Children. Clin. Infect. Dis. An Off. Publ. Infect. Dis. Soc Am. 65, S200-S219. doi: 10.1093/cid/cix664

Seale, A. C., Blencowe, H., Bianchi-Jassir, F., Embleton, N., Bassat, Q., Ordi, J., et al. (2017b). Stillbirth With Group B Streptococcus Disease Worldwide: Systematic Review and Meta-Analyses. Clin. Infect. Dis. An Off. Publ. Infect. Dis. Soc Am. 65, S125-S132. doi: 10.1093/cid/cix585

Senkovich, O., Ceaser, S., McGee, D. J., and Testerman, T. L. (2010). Unique Host Iron Utilization Mechanisms of Helicobacter Pylori Revealed With Iron-Deficient Chemically Defined Media. Infect. Immun. 78 (5), 1841-1849. doi: 10.1128/ IAI.01258-09

Shiroda, M., Aronoff, D. M., Gaddy, J. A., and Manning, S. D. (2020). The Impact of Lactobacillus on Group B Streptococcal Interactions With Cells of the Extraplacental Membranes. Microb. Pathog. 148, 104463. doi: 10.1016/ j.micpath.2020.104463

Shabayek, S., and Spellerberg, B. (2018). Group B Streptococcal Colonization, Molecular Characteristics, and Epidemiology. Front. Microbiol. 9, 437. doi: $10.3389 /$ fmicb.2018.00437

Sørensen, U. B. S., Poulsen, K., Ghezzo, C., Margarit, I., and Kilian, M. (2010). Emergence and Global Dissemination of Host-Specific Streptococcus Agalactiae Clones. MBio 1:e00178-10. doi: 10.1128/mBio.00178-10

Spaetgens, R., DeBella, K., Ma, D., Robertson, S., Mucenski, M., and Davies, H. D. (2002). Perinatal Antibiotic Usage and Changes in Colonization and Resistance Rates of Group B Streptococcus and Other Pathogens. Obstet. Gynecol. 100, 525-533. doi: 10.1016/s0029-7844(02)02068-9

Teatero, S., Ferrieri, P., Martin, I., Demczuk, W., McGeer, A., and Fittipaldi, N. (2017). Serotype Distribution, Population Structure, and Antimicrobial Resistance of Group B Streptococcus Strains Recovered From Colonized Pregnant Women. J. Clin. Microbiol. 55, 412-422. doi: 10.1128/JCM.01615-16

Tien, N., Ho, C.-M., Lin, H.-J., Shih, M.-C., Ho, M.-W., Lin, H.-C., et al. (2011). Multilocus Sequence Typing of Invasive Group B Streptococcus in Central Area of Taiwan. J. Microbiol. Immunol. Infect. 44, 430-434. doi: 10.1016/ j.jmii.2011.04.013

Trappetti, C., Potter, A. J., Paton, A. W., Oggioni, M. R., and Paton, J. C. (2011). LuxS Mediates Iron-Dependent Biofilm Formation, Competence, and Fratricide in Streptococcus Pneumoniae. Infect. Immun. 79, 4550-4558. doi: 10.1128/IAI.05644-11

Valenti, P., Rosa, L., Capobianco, D., Lepanto, M. S., Schiavi, E., Cutone, A., et al. (2018). Role of Lactobacilli and Lactoferrin in the Mucosal Cervicovaginal Defense. Front. Immunol. 9:376. doi: 10.3389/fimmu.2018.00376

Verani, J. R., McGee, L., Schrag, S. J.Division of Bacterial Diseases, National Center for Immunization and Respiratory Diseases and Centers for Disease Control and Prevention (CDC) (2010). Prevention of Perinatal Group B Streptococcal Disease-Revised Guidelines From CD. MMWR. Recomm. Rep. Morb. Mortal. Wkly Rep. Recomm. Rep. 59, 1-36.

Vornhagen, J., Adams Waldorf, K. M., and Rajagopal, L. (2017). Perinatal Group B Streptococcal Infections: Virulence Factors, Immunity, and Prevention Strategies. Trends Microbiol. 25, 919-931. doi: 10.1016/j.tim.2017.05.013 
Wang, P., Ma, Z., Tong, J., Zhao, R., Shi, W., Yu, S., et al. (2015). Serotype Distribution, Antimicrobial Resistance, and Molecular Characterization of Invasive Group B Streptococcus Isolates Recovered From Chinese Neonates. Int. J. Infect. Dis. IJID Off. Publ. Int. Soc Infect. Dis. 37, 115-118. doi: 10.1016/j.ijid.2015.06.019

Weinberg, E. D. (1975). Nutritional Immunity. Host's Attempt to Withold Iron From Microbial Invaders. JAMA 231, 39-41. doi: 10.1001/jama.1975.03240130021018

Winkelstein, J. A., Abramovitz, A. S., and Tomasz, A. (1980). Activation of C3 via the Alternative Complement Pathway Results in Fixation of $\mathrm{C} 3 \mathrm{~b}$ to the Pneumococcal Cell Wall. J. Immunol. 124, 2502-2506.

Conflict of Interest: The authors declare that the research was conducted in the absence of any commercial or financial relationships that could be construed as a potential conflict of interest.
Publisher's Note: All claims expressed in this article are solely those of the authors and do not necessarily represent those of their affiliated organizations, or those of the publisher, the editors and the reviewers. Any product that may be evaluated in this article, or claim that may be made by its manufacturer, is not guaranteed or endorsed by the publisher.

Copyright (c) 2021 Lu, Guevara, Francis, Spicer, Moore, Chambers, Craft, Manning, Townsend and Gaddy. This is an open-access article distributed under the terms of the Creative Commons Attribution License (CC BY). The use, distribution or reproduction in other forums is permitted, provided the original author(s) and the copyright owner(s) are credited and that the original publication in this journal is cited, in accordance with accepted academic practice. No use, distribution or reproduction is permitted which does not comply with these terms. 